\title{
Using Social Media in Diabetes: Best Practice from Ahmedabad
}

\author{
Rutul Gokalani ${ }^{1}$ \\ ${ }^{1}$ AHC DiaCare-Diabetes Clinic, Ahmedabad, Gujarat, India
}

J Soc Health Diab 2019;7:36

This letter highlights the use of social media in the treatment of diabetes, and the uses of technology that has proven beneficial in treating lifelong condition.

As the world is changing, traditional health care services have been replaced by the new era of technology. Patients use Internet to stay in touch with others and also use it to share clinical information and receive the required support. In my daily practice, I have created two separate WhatsApp groups for Type 1 and Type 2 diabetes. The WhatsApp application is used as an alternative treatment to regular clinical visit. The group is open on weekends, where participants are free to ask their questions, inform their blood glucose value, or ask for any suggestions related to the disease. Persons with diabetes who are not added into the group prefer to text me their fasting and prandial values personally.

To my part, I respond to their queries promptly and titrate their medicine/insulin dosage if needed. In case the condition is deteriorating, I advise them to visit clinic. Also, laboratory investigations to be performed are informed prior to the visit, thus saving time. Facebook has helped most of my adult patients living with Type 1 diabetes. I use Facebook for posting information related to diabetes and
Address for correspondence Rutul Gokalani, MD, AHC DiaCare-Diabetes Clinic, Next to Saibaba Mandir, Ghatlodiya, Ahmedabad-380061, Gujarat, India (e-mail: ruturaj.9560@gmail.com).

technology, physical activities, or calorie intake. These updates help as well as empower the patients to fight against this chronic disease.

Facebook is used as a tool for education. This wise use of technology allows patients to gain knowledge of the disease and be in constant contact with the treating physician. I try to use this platform to treat and educate as many patients as possible. Also, diabetes is a disease where the patient has to keep an eye on food intake, mental status, and exercise, all under expert advice. All this can be done through social media now.

Using the aforementioned platforms since the past one year has shown improvement in diabetes control, similar to regular clinical visits. The rising burden of diabetes has affected the economic state of individuals and families as well, as the cost of treatment is high and is mostly individually borne. If the physician tries to lessen the burden by use of technology, this can prove to be a boon for the society.

\section{Conflict of Interest \\ None declared.}

License terms 